\title{
1 Principles for coding associative memories in a compact
}

\section{neural network}

3 Chrisitian O. Pritz, Eyal Itskovits, Eduard Bokman, Rotem Ruach, Vladimir Gritsenko, Tal

4 Nelken, Mai Menasherof, Aharon Azulay, and Alon Zaslaver*

5

6 Department of Genetics, Silberman Institute of Life Science, Edmond J. Safra Campus, The

7 Hebrew University of Jerusalem, Jerusalem 9190401, Israel

8 *Corresponding author: Alon Zaslaver alonzas@mail.huji.ac.il

9

\section{Summary}

11 A major goal in neuroscience is to elucidate the principles by which memories are stored in a

12 neural network. Here, we have systematically studied how the four types of associative

13 memories (short- and long-term memories, each formed using positive and negative

14 associations) are encoded within the compact neural network of $C$. elegans worms.

15 Interestingly, short-term, but not long-term, memories are evident in the sensory system.

16 Long-term memories are relegated to inner layers of the network, allowing the sensory system

17 to resume innate functionality. Furthermore, a small set of sensory neurons is allocated for

18 coding short-term memories, a design that can increase memory capacity and limit non-innate

19 behavioral responses. Notably, individual sensory neurons may code for the conditioned

20 stimulus or the experience valence. Interneurons integrate these information to modulate

21 animal behavior upon memory reactivation. This comprehensive study reveals basic principles

22 by which memories are encoded within a neural network, and highlights the central roles of

23 sensory neurons in memory formation. 


\section{Introduction}

Learning and memory processes are presumably universal in the animal kingdom,

26 forming the basis for adaptive behavior. An intriguing form of these behavioral adaptations is

27 known as associative learning, where a link between two unrelated cues is formed. The

28 famous pavlovian dogs set a classical example: These dogs were trained to associate the

29 sound of a bell (the conditioned stimulus, CS) with food (unconditioned stimulus, US).

30 Consequently, the mere sound of the bell prompted the dogs to salivate in expectation for their

31 meal (Pavlov, 1910).

To synthesize a productive associative memory that elicits an adaptive response upon

33 future encounter with the CS, both the CS and the US must be encoded in the neural system.

34 Moreover, their encoding needs to be logically integrated such that the behavioral response

35 will match the expected valence that the CS predicts (Josselyn and Tonegawa, 2020).

36 Whether the CS was associated with a positive or negative experience, this valence remains

37 associated with the CS.

Animals have come up with different strategies for encoding associative memories. In

39 flies, olfactory associative learning is centralized in the mushroom body, where both the

40 valence of the US and the CS odorant are encoded (Widmann et al., 2018). In contrast,

41 mammalian brains are thought to encode associative memories in a decentralized fashion

42 where interconnected areas, distributed throughout the entire brain, link up to encode memory

43 traces. For example, associative fear memories are thought to be distributed among the

44 amygdala that encodes the valence, the hippocampus which encodes the context, and the

45 cortical neurons which provide the specific sensory information (Josselyn and Tonegawa,

46 2020). 
Furthermore, sensory neurons proved to play intriguing roles in formation of

associative memories. Their learning-induced neuroplasticity was observed across various

49 sensory modalities (e.g. olfactory, gustatory, auditory, and visual), and is thought to confer

50 improved detection and enhanced attention towards important cues encountered in the past

51 (Åhs et al., 2013; McGann, 2015).

53 first to identify the brain regions, and ultimately the individual neurons, that participate in these

54 processes. To this end, Caenorhabditis elegans worms offer an appealing research system.

55 Their compact nervous system consists of 300 neurons, and a detailed blueprint of all the

56 chemical and electrical connections is available (White et al., 1986; Cook et al., 2019; Witvliet

57 et al., 2020). Moreover, the number and the identity of the neurons is invariant and individual

58 neurons can be unambiguously identified according to their position and anatomy across

59 different individuals. Crucially, though equipped with a small neural network, $C$. elegans worms

60 can form both associative and non-associative memories (Ardiel and Rankin, 2010; Sasakura

61 and Mori, 2013). As in higher organisms, these memories can be classified into short- and

62 long-term memories, which in worms last for a couple of hours or days, respectively (Kauffman

63 et al., 2010; Amano and Maruyama, 2011). Whole-brain functional imaging enables measuring activity from large volumes in 65 animals that express activity-dependent fluorescent markers (Ahrens et al., 2013; Aimon et al., 66 2019; Voleti et al., 2019). In C. elegans, advanced microscopy techniques allow imaging 67 neural dynamics of the entire network with cellular resolution in both stationary and 68 freely-behaving animals (Schrödel et al., 2013; Kato et al., 2015; Nguyen et al., 2016; 69 Venkatachalam et al., 2016; Toyoshima et al., 2020). 
71 addressing intriguing questions that were hitherto considered mainly based on theoretical

72 grounds. For example, how does plasticity of individual sensory neurons code and integrate

73 the stimulus and the valence of the experience? How many neural resources are required to

74 form an associative memory, and more fundamentally, are there general principles by which

75 associative memories are encoded within a neural network?

77 (positive/negative associations, each formed as a short- or long-term memory) are encoded

78 within the compact neural network of $C$. elegans. Short-term, but not long-term, memories are

79 evident in the sensory system of the animal. Moreover, a limited set of sensory neurons codes

80 each of the short-term memories where individual neurons may code the conditioned stimulus

81 or the valence of the experience. The downstream interneuron layer codes both short- and

82 long-term memories, and individual interneurons integrate both stimulus and valence

83 information to dictate behavioral outputs.

87 memories (Fig. 1A). Building on existing protocols (Bargmann, Hartwieg and Horvitz, 1993;

88 Kauffman et al., 2011), we trained C. elegans worms to form each of the four types of

89 associative memories: short-term aversive (STAV), short-term appetitive (STAP), long-term

90 aversive (LTAV), and long-term appetitive (LTAP). Notably, we used the same CS (the odorant

91 butanone) for all training paradigms. This allowed us to extract memory traces that are unique

92 to the training paradigm and that are independent of the specific CS used. 
To form positive (appetitive) or negative (aversive) associations, we exposed the

94 worms to butanone in the presence or the absence of food, respectively. To form short-term

95 memories, for which behavioral changes last for up to 2 hours (Kauffman et al., 2010), we

96 trained the worms for 1 hour and assayed the animals within 30 minutes following the training

97 period. For long-term memories, which typically last for $1-2$ days (>10\% of the worms'

98 lifespan), we used repetitive-training regimes that lasted for $\sim 12$ hours and then assayed the

99 animals 14 hours post the training period (Fig. 1A, Methods). In parallel to the trained animals,

100 we always included mock-trained animals (animals that underwent training in the absence of

101 the CS butanone) as well as naive animals, which were left untreated, as controls.

103 attraction of trained animals to the CS butanone (Fig. 1B). For this, we used a standard

104 two-choice assay, where worms were free to choose between the CS and an alternative

105 attractant, diacetyl, and calculated the Choice Index $(\mathrm{Cl})$, which provides a quantitative

106 measure for the animals' preference towards the CS (Fig. 1B, suppl. Fig. S1A).

108 butanone than mock-trained or naive animals (Fig. 1C-D). Similarly, worms trained to form

109 negative associations, were significantly less attracted to butanone when compared to

110 mock-trained or naive animals. These behavioral changes were evident in both short- and the

111 long-term training paradigms. Similar results were also obtained when using ethanol (butanone

112 diluent) instead of diacetyl as the alternative choice (suppl. Fig. S1B).

113 We next quantified the explicit effects of the CS (butanone) and the US

114 (starvation/appetitive experience) on the behavioral output. For this, we used the Clvalues to

115 compute the Learning Index (LI), the difference between the choice indices of the different

116 experimental groups within a training paradigm (Fig. 1B and Methods). The treatment itself

$117\left(\mathrm{Cl}_{\text {mock }}-\mathrm{Cl}_{\text {naive }}\right)$ had negligible effects, except for a mild effect noted following the STAP- training 
118 protocol (Fig 1D). However, in all paradigms, significant changes in choice (both training

$119\left(\mathrm{Cl}_{\text {trained }}-\mathrm{Cl}_{\text {naive }}\right)$ and stimulus $\left(\mathrm{Cl}_{\text {trained }}-\mathrm{Cl}_{\text {mock }}\right)$-specific $)$ indicated that the modulated choice of

120 butanone was due to the presence of butanone during training. Furthermore, the change in the

121 choice corresponded to the valence of the experience: appetitively-trained animals increased

122 their choice of butanone, while aversively-trained animals decreased their choice. Thus, the

123 change in choice behavior is dependent on both the stimulus and the valence of the

124 experience.

125 Taken together, using the same CS butanone, we could robustly form all the four types

126 of associative memories: positive (appetitive) associative memories which increased attraction

127 towards butanone, and negative (starvation) associative memories which decreased this

128 attraction. Both negative and positive experiences could be used to form short- and long-term

129 memories. 


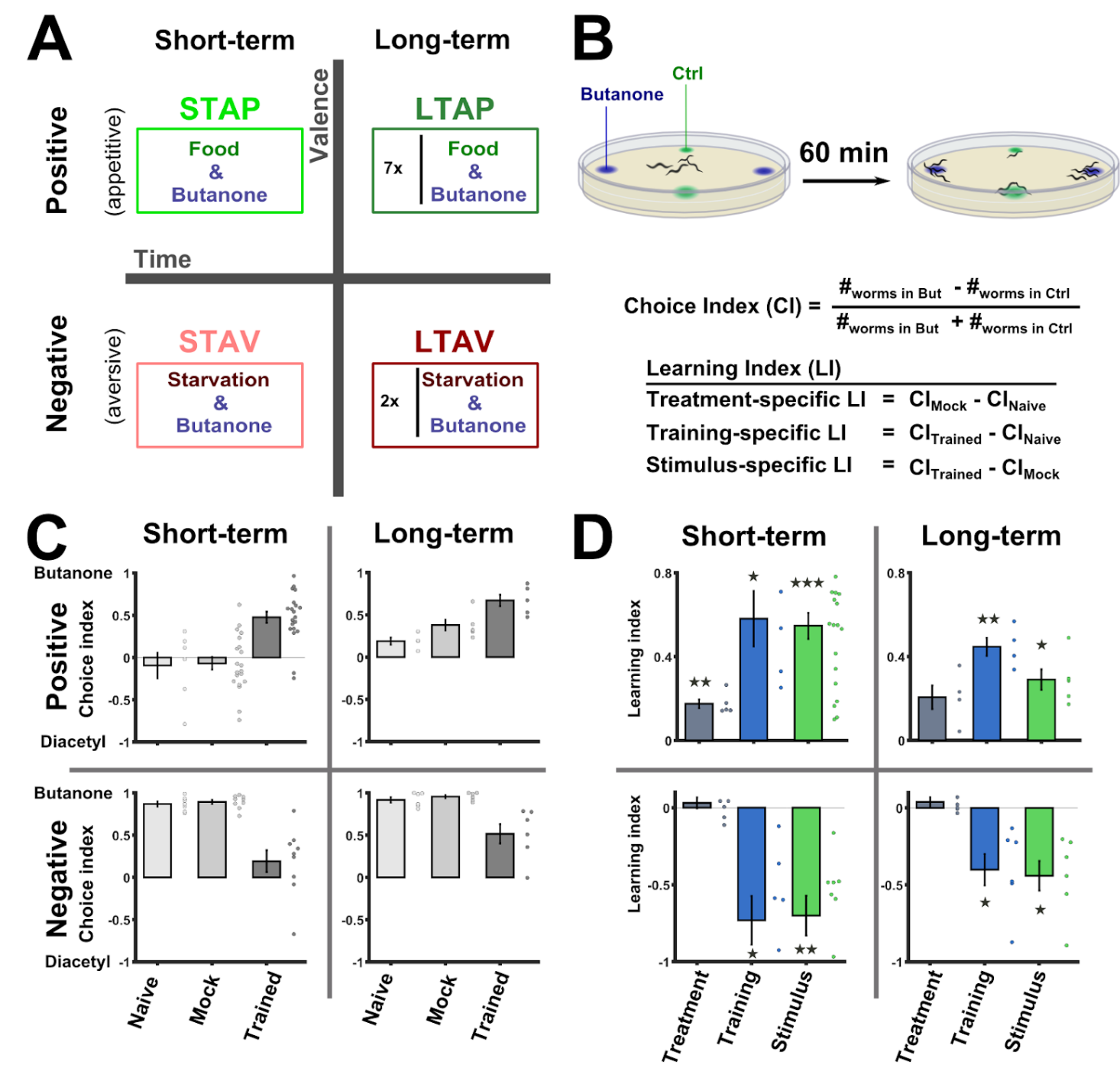

130 Fig. 1. Training paradigms that form robust associative memories.

131 (A) Worms were trained to form each of the four types of associative memories: Short- and 132 long- term memories (denoted along the horizontal axis), each trained using a positive or 133 negative unconditioned stimulus (vertical axis). Notably, the same conditioned stimulus, 134 butanone, was used for all types of memory. STAP, short-term appetitive; LTAP, long-term 135 appetitive; STAV, short-term aversive; LTAV, long-term aversive.

136 (B) A two-choice assay was used to quantify animals' preference towards the conditioned 137 stimulus butanone (against the alternative attractive choice, diacetyl). Scoring the number of 138 worms reaching each of the choices provided the Choice Index $(\mathrm{Cl})$, which ranges from -1 139 (denoting complete aversion to the CS) to +1 (full attraction). Choice tests for positively- and 140 negatively- trained animals differed in concentrations and layout (see suppl. Fig. S1A) because 141 of valence-specific effects on choice behavior (suppl. Fig. S2). Learning indices (Lls), 142 calculated based on these Cls, show the treament-, stimulus-, and training- specific effects on 143 the animals' choice (suppl. Fig. S3).

144 (C) Choice index values as scored following the behavioral choice assays. Positively-trained 
145 animals increased attraction while negatively-trained animals reduced attraction towards

146 butanone.

147 (D) Learning indices calculated according to the equations provided in (B) on the data shown

148 in (C). Significant stimulus- and training-specific Lls in all paradigms indicate

149 experience-dependent modulation of behavior that is based on stimulus and valence.

150 Strikingly, Learning indices were calculated by comparing experiments performed on the same

151 day. (suppl. Fig. S4 and Methods). Experimental repeats (C\&D) were performed on different

152 days and range between 4-21. Each experimental repeat is the average of three assay plates,

153 each scoring 100-150 worms. Error bars indicate SEM. ${ }^{*} p<0.05,{ }^{* *} p<0.01,{ }^{* * *} p<0.001$

154 (one-sample t-test, FDR corrected; significant differences from the zero LI values).

Memory formation is typically associated with changes in synaptic strength among

157 neurons that participate in encoding the memory (Byrne, Castellucci and Kandel, 1978;

158 Hopfield, 1982). As a result, activity of these memory-storing neurons may be modulated when

159 compared to their activity in naive or mock-trained animals. In that respect, quantifying calcium

160 dynamics within individual neurons is a powerful technique to extract fine neural dynamics

161 (Kerr et al., 2000; Ahrens et al., 2013).

We begin by analyzing neuroplasticity of the sensory system, naturally focusing on the

163 chemosensory neurons. To measure response dynamics in individual chemosensory neurons,

164 we imaged a transgenic strain that expresses the genetically encoded calcium indicator,

165 GCaMP, in virtually all chemosensory neurons (Iwanir et al., 2019). We restrained the animals

166 in a microfluidic device (Chronis, Zimmer and Bargmann, 2007), and used a fast-scanning

167 confocal system to collect fluorescent image stacks of all chemosensory neurons during

168 exposure to the CS butanone (Fig. 2A).

Naive C. elegans worms showed robust innate responses in AWA, ASH, AWC, AWB,

170 ASJ, and URX sensory neurons towards exposure or removal of butanone (Fig. 2A and suppl.

171 Fig. S5, S6 and in agreement with previous reports (Bargmann, Hartwieg and Horvitz, 1993;

172 Kato et al., 2014). However, following training and memory formation, response activity of 
173 merely four neuron types, namely, AWC, ASH, AWA and ASI, was markedly modulated (Fig.

174 2B-G,E, suppl. Figs. S7, S8). These neural modulations were observed only in the short-term

175 memory paradigms, and none of these changes persisted in the long-lasting memories (see

176 suppl. Fig. S9, except for the ASI neurons, whose modulated dynamics appeared only in half of

177 the population, suppl. Fig. S7).

179 only in the short-term paradigms (Fig. 2B-C, suppl. Fig. S8 A-D). While the AWC ${ }^{\text {OFF neuron }}$

180 exhibited robust innate responses in naive animals, its responses were significantly elevated

181 following formation of short-term memories (suppl. Fig. S10). The AWC ${ }^{\mathrm{N}}$ neuron lacked

182 activity responses in naive and mock-trained animals, but gained marked responses following

183 formation of short-term memories (suppl. Fig. S10). Notably, these observations were robustly

184 replicated in an additional and independent strain that expresses GCaMP exclusively in the

$185 \mathrm{AWC}^{\mathrm{ON}}$ and $\mathrm{AWC} \mathrm{C}^{\mathrm{OFF}}$ neurons, and which allowed unambiguously identifying each of these

186 neurons (suppl. Fig. S10 and see Methods). Moreover, the gained responses in STAP-trained

187 animals were dependent on intact synaptic activity (but not on neuropeptide signaling, suppl.

188 fig. S11), indicating that active network dynamics is required for modulating AWC ${ }^{\mathrm{ON}}$ responses.

190 trained animals did not significantly differ (suppl. Fig. S10 A\&B), suggesting that these neurons

191 do not distinguish between the valence (the appetitive or aversive experience) of the memory.

192 In contrast, the responses of the trained animals were significantly higher than their matched

193 mock-trained controls (which were not exposed to the CS, Fig. 2 B \& C bar graphs, suppl. Fig.

194 S10 A\&B), suggesting that these neurons may be coding the conditioned stimulus (suppl. fig.

195 S3), a finding that is in line with previous reports (Cho et al., 2016). 
198 responses of the matched mock-control animals, suggesting that the ASH neurons may be

199 coding the stimulus. However, since the increased response was observed following the

200 negative training only (Fig. 2D), it is also possible that these neurons code for the valence

201 component of the memory.

203 positive and the negative short-training paradigms (Fig. 2E). These responses were not

204 different from their matched mock-trained controls, suggesting that the AWA neurons may be

205 coding the experience (valence) rather than the stimulus (Fig. 2E, suppl. Fig. S3).

207 quantified the preference of trained animals, defective in each of these individual neuron

208 types, towards the CS (as shown in Fig. 1A). Animals with genetically-ablated AWC neurons

209 lost their learning and memory abilities as indicated by the near zero LI values for both the

210 positive and the negative training paradigms (suppl. Fig. 12 A-B). Animals with

211 functionally-defective AWA neurons $\left(\right.$ odr $\left.-7^{-1}\right)$, showed impaired learning abilities following

212 negative training only, while animals with genetically-ablated $\mathrm{ASH}$ neurons were not

213 significantly imapired (suppl. Fig. 12 A-B). These results indicate that neurons showing

214 modulated activity in memory retrieval are indeed critical for memory formation or memory retrieval (or both) processes.

216 encoded within a limited set of sensory neurons. Moreover, individual sensory neurons may

217 code for either the stimulus or the valence (experience), or possibly like the ASH neurons,

218 integrate the stimulus and the valence information. 
bioRxiv preprint doi: https://doi.org/10.1101/2020.06.20.162818; this version posted June 21, 2020. The copyright holder for this preprint (which was not certified by peer review) is the author/funder, who has granted bioRxiv a license to display the preprint in perpetuity. It is made available under aCC-BY-ND 4.0 International license.
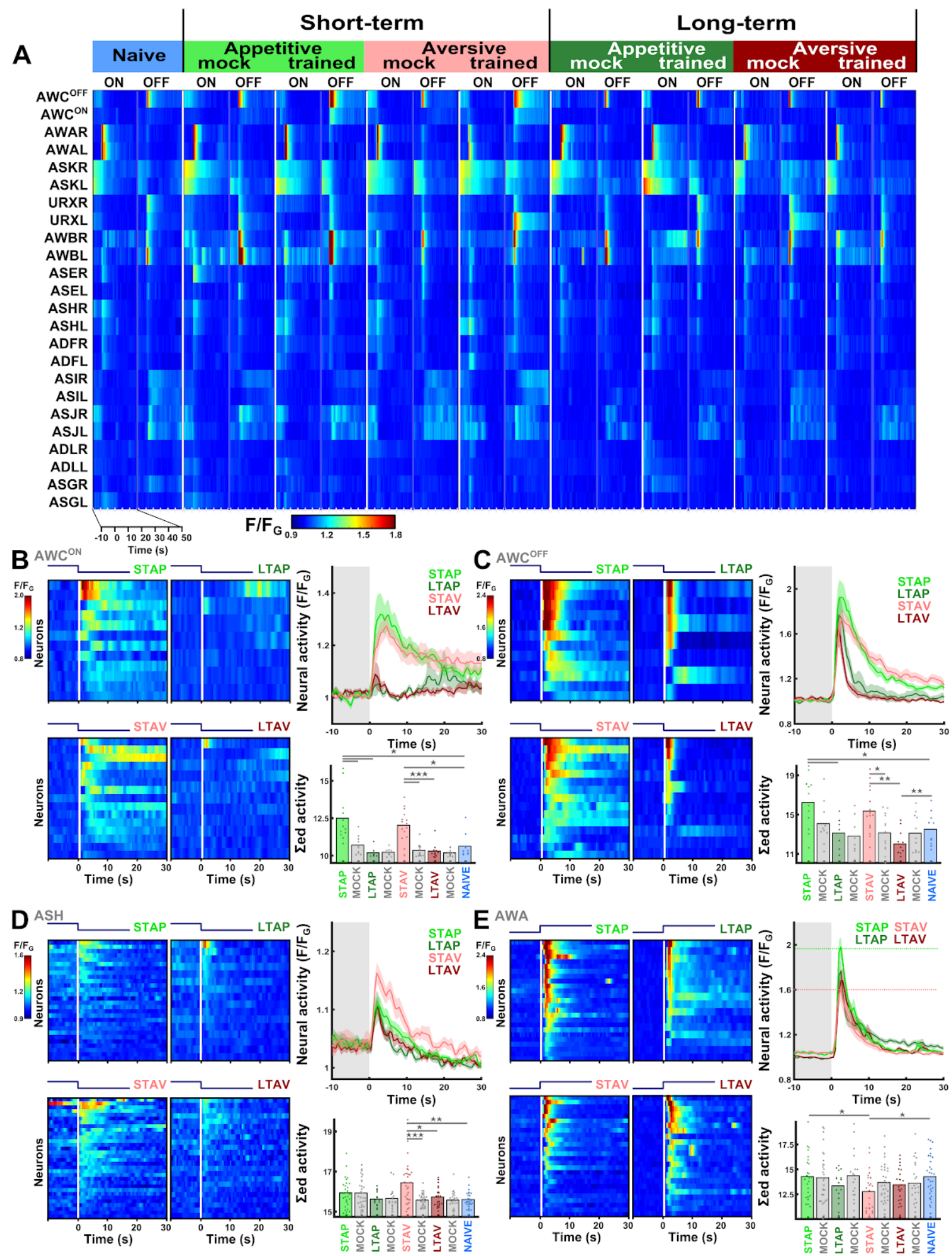

219 Figure 2. Short term, but not long-term, memories are evident at the sensory layer.

220 (A) Neural dynamics following exposure to (ON) or removal of (OFF) the conditioned stimulus

221 butanone. These activity dynamics were systematically measured in animals trained in the four 
222 associative-memory paradigms (STAP, STAV, LTAP, LTAV, Fig. 1A), as well as in their matched

223 mock-trained and naive control animals. 9-17 animals were imaged per group (column),

224 resulting in coverage of 2-17 per neuronal trace (median=13).

225 (B-E) The sensory neurons, AWC ${ }^{\mathrm{ON}}(\mathrm{B}), \mathrm{AWC}^{\mathrm{OFF}}(\mathrm{C}), \mathrm{ASH}(\mathrm{D})$, and AWA (E) show significantly 226 differential activity following formation of short-term memories. Heat maps denote activities of 227 individual neurons in each of the training paradigms. Horizontal white line at $t=0$ denotes the 228 time of stimulus exchange. In the mean line plots, the different colors denote the trained 229 animals in a given paradigm. Shaded gray background indicates butanone exposure, and 230 shaded area around the mean activity indicates standard error of mean. Bar graphs represent 231 the summed neuronal activity post the stimulus switch. Blue, naive worms; Gray, mock-trained 232 worms. Next to each bar, the groups that show significant differences are indicated. ${ }^{*} p<0.05$, $233{ }^{* *} \mathrm{p}<0.01,{ }^{* * *} \mathrm{p}<0.001$ (t-test, FDR corrected).

234 (B) In the AWC ${ }^{O N}$ neuron, STAV- and STAP-trained animals showed similar elevated 235 responses, which were significantly higher than their matched mock-trained controls (bar 236 graphs and suppl. Fig. S8), suggesting stimulus-specific encoding.

237 (C) In the AWC ${ }^{\text {OFF }}$ neuron, responses following STAV- and STAP-training were significnatly 238 increased. STAV-trained animals exhibited significantly higher responses when compared to 239 the mock-trained animals (bar graphs and suppl. Fig. S8), suggesting stimulus-specific coding.

240 (D) In ASH neurons, activity is increased after STAV training only. Note the significant 241 difference from the mock-trained controls. ASHL and ASHR showed similar response 242 dynamics, so shown are their averaged activities.

243 (E) STAV-trained animals showed significantly higher responses than STAP-trained animals in 244 AWA neurons, suggesting valence-specific differences. Horizontal dotted lines in line graphs 245 highlight amplitude differences between STAV and STAP. AWAL and AWAR showed similar 246 response dynamics, so presented are their averaged activities.

249 memories (both LTAP and LTAV, fig. 2A and suppl. Fig. S9), we speculated that long-term 250 memory traces may have shifted to the downstream interneuron layer. Since short-term 251 memories showed modulated activity primarily in AWC, ASH and AWA neurons (Fig. 2), we 252 studied their main shared downstream interneurons, namely, AIA, RIA and AIB (Fig. 3, suppl. 253 Fig. S13 and S14). 

interneurons. While all three neurons showed innate responses to butanone in naive animals, both AIA and RIA (but not AIB) showed significantly altered responses to butanone following

257 memory formation (Fig. 3). As previously reported for AIA and RIA (Chalasani et al., 2010;

258 Hendricks et al., 2012), these modulated dynamics were observed in the neurites rather than

259 in the cell soma (Fig 3 B \& C).

261 and mock-trained controls (Fig. 3D-F), suggesting that the AIA neurons may be coding the

262 conditioned stimulus. The enhanced response in LTAV-trained animals was also significantly

263 higher than the response found in LTAP-trained animals, suggesting that these neurons may

264 also code the negative experience. Thus, AIA neurons may be integrating information of both

265 the stimulus and the valence.

267 proprioceptive feedback, that indicates the animal's head position, together with sensory inputs

268 (Hendricks et al., 2012; Ouellette et al., 2018). Remarkably, following long-term training

269 paradigms, the baseline activity of the RIA neurons was elevated before the exposure to the

270 stimulus (compare heat maps from Fig. 3G to heat maps in suppl. Fig. S13B). Following

271 stimulus presentation, only in LTAV-trained animals activity in the dorsal neurite was

272 maintained at high levels, while in all other training conditions activity markedly decreased (Fig.

273 3G-I). Similar, though less pronounced, results were obtained by imaging the ventral neurite of

274 the neuron (suppl. Fig. S13E \& G), where LTAV-trained animals showed highest post-stimulus

275 activity. The differential activity between LTAV and LTAP training, and the fact that

276 LTAV-trained animals showed higher activity than mock-control animals (Fig. 3F), suggest that

277 the RIA neurons may encode the valence and the stimulus components of the memory. 

al., 2012; Ouellette et al., 2018). These inputs were evident in both the ventral and the dorsal compartments of the neurite following stimulus removal (Fig. 3B, J, K, \& L). However, the sensory-evoked activity was significantly prolonged in STAV-trained animals (compared to STAP- and mock- trained animals, Fig 3F and suppl Fig. S13D). This suggests that, in addition to long-term memories, the RIA interneurons integrate stimulus- and valence- sensory

284 information stored in short-term memory. As RIA neurons directly connect to motor outputs, 285 integrated information of stored and newly-sensed inputs can be efficiently mediated to 286 respective behavioral outputs. downstream of the above studied interneurons (Chalfie, 1984). We therefore studied whether

289 activity dynamics in command neurons is also modulated following memory acquisition. For

290 this, we used a transgenic strain that expresses GCaMP pan-neuronally (Nguyen et al 2017),

291 from which we could reliably extract activities of the backward-motion promoting neurons AVA,

292 AVE, AVD as well as from the forward-motion promoting neuron AVB (Fig. 3A and suppl. Fig.

293 S14). Indeed, activities among the backward command neurons were highly correlated, and

294 anti-correlated to the activity of the forward command neuron (suppl. Fig. S14). Based on the

295 activity dynamics of the AVA neurons, whose response was most prominent, we could not

296 detect differences in the response dynamics following the various training paradigms (suppl.

297 Fig. S14B-E), suggesting that these neurons do not participate (or at the very least, play a 298 minor role) in memory encoding.

300 modulated by the valence and the stimulus in both short- and long-term memories. Thus, 301 individual interneurons may serve as integrating hubs for the encoded memory information. 
bioRxiv preprint doi: https://doi.org/10.1101/2020.06.20.162818; this version posted June 21, 2020. The copyright holder for this preprint (which was not certified by peer review) is the author/funder, who has granted bioRxiv a license to display the preprint in perpetuity. It is made available under aCC-BY-ND 4.0 International license.

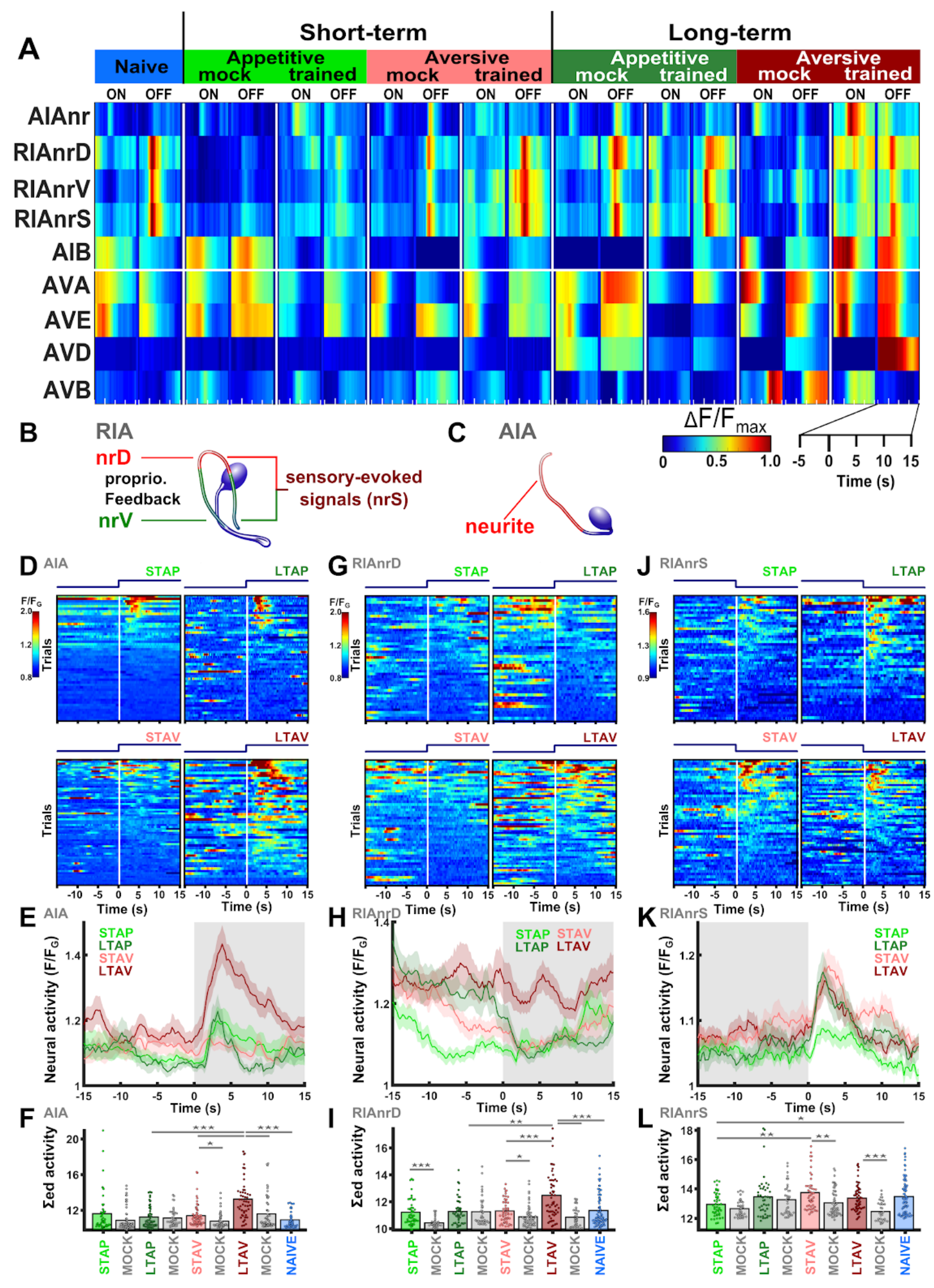

303 Figure 3. Interneurons integrate valence and stimulus information of short- and long304 term memories. 
305 (A) Activity profiles of inter- and command- neurons following memory formation according to 306 each of the four training paradigms (STAP, STAV, LTAP, LTAV, Fig. 1A). Butanone presentation

$307(\mathrm{ON})$ or removal (OFF) was at $\mathrm{t}=0$. ' $\mathrm{nr}$ ' next to the neuron name denotes imaging Ca dynamics 308 from the neurite. D, dorsal; V, ventral; S, stimulus-evoked signal. Each neural trace is the mean 309 of at least four repeats. 9-15 individual animals were imaged.

310 (B) Activity of the RIA neurons was measured in the dorsal (RIAnrD, red) and the ventral 311 (RIAnrV, green) compartments of the neurite. The sensory-evoked signal (RIAnrS) was 312 extracted as the overlap in signal from both the dorsal and the ventral compartments upon 313 stimulus removal.

314 (C) Activity in the AIA neurons was measured from the neurite (red region).

315 (D) Heat maps, (E) line plots, and (F) bar graphs depicting activity in the AIA neurite (AIAnr). 316 Note the increased activity following LTAV training and the differential activity following LTAP

317 and associated mock-trained controls (stimulus- and valence specific differences).

318 (G) Heat maps, (H) line plots, and (I) bar graphs depicting activity in the RIA dorsal neurite 319 (RIAnrD). LTAV-trained animals show differential activity when compared to other training 320 paradigms or their matched mock-trained controls, suggesting for stimulus and 321 valence-specific differences (see also line graph \& suppl. Fig. S13).

322 (J) Heatmaps, (K) line plots, and (L) bar graphs depicting activity in the RIA dorsal neurite 323 (RIAnrD). Note the increased activity in STAV- compared to STAP- training and mock-trained 324 controls (stimulus and valence-specific differences, also see suppl Fig. S13).

325 Line graphs: colors denote the trained animals in each of the training paradigms. Shaded 326 areas in the line plots indicate SEM. Bar graphs represent the summed neuronal activity post 327 stimulus exchange. ${ }^{*} p<0.05,{ }^{* *} p<0.01,{ }^{* * *} p<0.001$, t-test, FDR corrected.

331 dynamics of sensory- and inter- neurons (Figs. 2-3), which in turn, modulates animal attraction

332 towards the training stimulus (Fig. 1). We next asked what are the fine locomotive changes

333 that follow memory acquisition and which underlie the modulated attraction towards the CS.

334 For this, we imaged trained worms during chemotaxis towards butanone (Fig. 4A) using a

335 multi-animal tracking system that extracts key locomotion parameters from over a hundred 336 worms at a time (Itskovits et al., 2017). As expected, aversively-trained animals were 337 significantly slower to arrive at the butanone target point when compared to positively-trained 
338 or naive animals (Fig. 4B, consistent with the chemotaxis results shown in Fig. 1C). However,

339 positive training yielded no improvement as both trained and mock-trained controls were

340 slower to leave the starting point area, presumably due to the specific technicalities of the

341 assay (see suppl. Fig. S15C).

343 following aversive training, we analyzed key chemotaxis properties, including speed, turning

344 frequency, and the directionality of the animals in relation to the CS endpoint (Fig. $4 \mathrm{C}-\mathrm{H}$ and

345 suppl. Fig. S15). Notably, negatively-trained animals were significantly slower during

346 chemotaxis (compared to naive, mock-trained and positively-trained worms, Fig. 4 C,D and

347 suppl. Fig. S15D-F). Moreover, the reduced speed was irrespective of the distance from the

348 CS target point.

350 reversal rates when reaching the proximity of the CS (compared to naive, mock-trained

351 controls, and positively-trained animals, Fig. 4E\&F, suppl. Fig. S15G). Furthermore, the

352 trajectories of LTAV-trained animals became less directed towards the target CS as reflected

353 by their increased deviation angle (Fig. 4G\&H, suppl. Fig. $\mathrm{S} 15 \mathrm{H}$ ), indicating their aversion

354 once approaching close to the CS.

356 parameters, including speed, turning frequency, and directionality towards the target

357 containing the aversive conditioned stimulus. The combined effect of these behavioral

358 modulations can explain the diminished attraction towards the CS following formation of 359 negative associations. 
bioRxiv preprint doi: https://doi.org/10.1101/2020.06.20.162818; this version posted June 21, 2020. The copyright holder for this preprint (which was not certified by peer review) is the author/funder, who has granted bioRxiv a license to display the preprint in perpetuity. It is made available under ACC-BY-ND 4.0 International license.
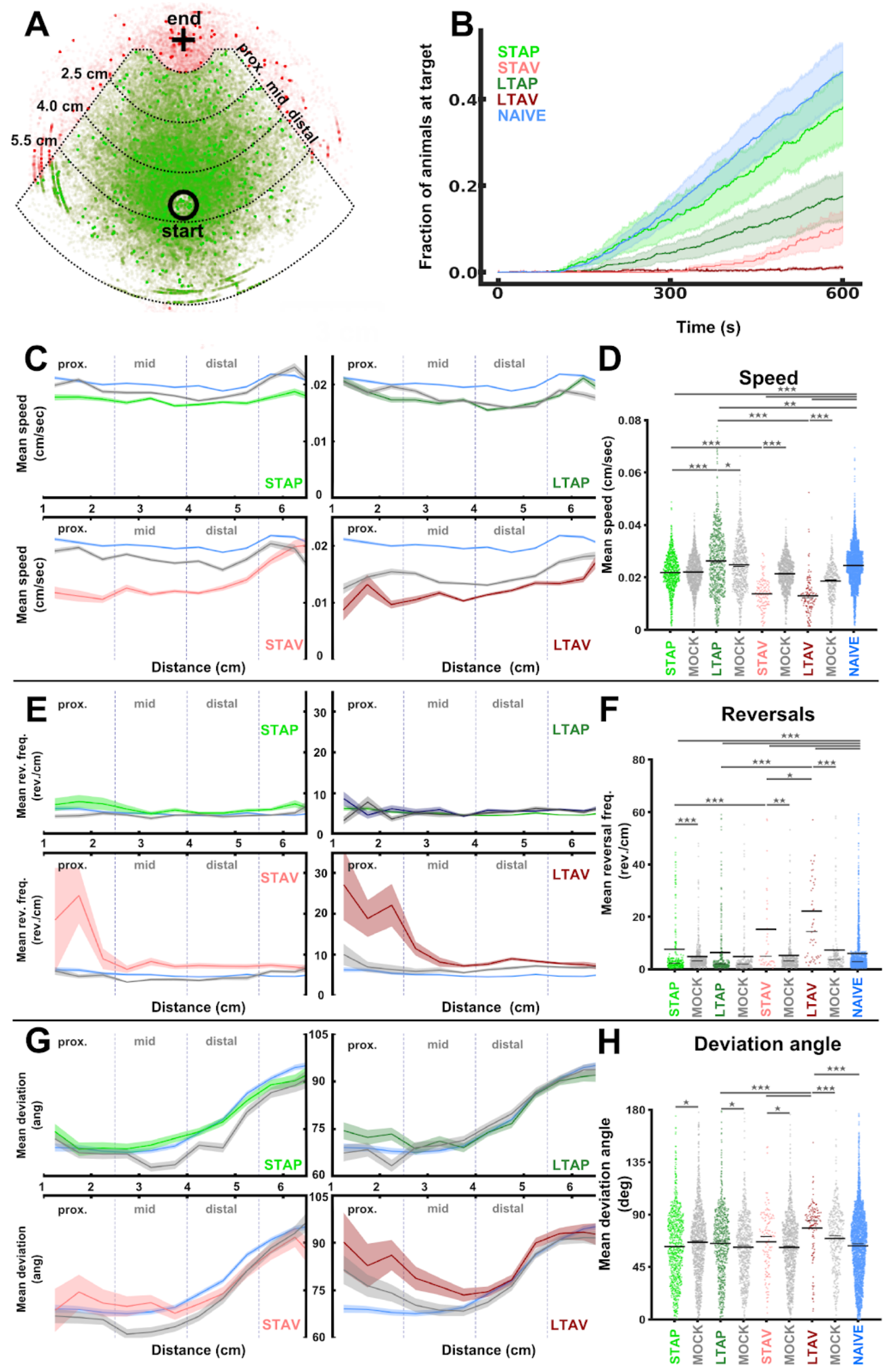


\section{Figure 4. Memory-induced neural changes modify fine behavioral outputs.}

362 (A) A layout of the one-choice chemotaxis assay. Butanone was applied on a chemotaxis plate 363 (marked as + region), and $\sim 100$ worms, placed $5 \mathrm{~cm}$ away (black circle), were imaged during 36410 minutes of chemotaxis. For analysis purposes, the arena was divided into three radial 365 segments: proximal, mid., and distal, spaced $1.5 \mathrm{~cm}$ apart. Tracks bordering the plate rim were 366 excluded (red points). All other tracks (green) were collected to extract locomotion parameters, 367 as shown in panels $\mathrm{C}-\mathrm{H}$.

368 (B) Temporal accumulation of animals at the butanone target region (marked + in panel A). 369 Positively-trained and naive animals reached the target point faster than aversively-trained 370 animals. 4-11 independent experimental repeats for each of the different conditions (21 for 371 naive). Shaded areas denote standard error (estimated by bootstrap analysis).

372 (C) Mean locomotion speed as a function of the distance from the target point.

373 (D) Mean locomotion speed in the proximal zone. Note that speed is significantly reduced in 374 aversively trained animals.

375 (E) Mean reversal rates as a function of the distance from the target point for each of the four 376 training paradigms.

377 (F) Mean reversal rates in the proximal zone only. Reversals are significantly increased in 378 aversively trained animals.

379 (G) Deviation angles of animal's trajectories from the target CS point. The deviation angles are 380 plotted as a function of the animal's distance from the target for each of the four training 381 paradigms.

382 (H) Deviation angles in the proximal zone only. Note that the deviation angle is significantly 383 increased in LTAV-trained animals.

384 In D,F,H: Asterisks and notations indicate significant differences. Error bars indicate SEM. $385{ }^{*} p<0.05,{ }^{* *} p<0.01,{ }^{* * *} p<0.001$ (rank sum test, FDR corrected). each of the four forms of associative memories: short- and long- term memories, each coded

391 using a positive and a negative association. Importantly, the use of the same conditioned 
392 stimulus (CS, butanone) in all memory paradigms allowed us to extract the individual neurons

393 that exclusively code the experience valence (positive or negative), the conditioned stimulus,

394 or both.

396 dynamics of sensory neurons towards the CS (Fig. 2). We observed significant changes in at

397 least three sensory neuron types following formation of short-term memories. The AWC

398 neurons showed clear modulated responses in a stimulus-specific manner (Fig. 2B-C), in line

399 with previous findings showing that the AWC neurons represent sensory history by shifting

400 their susceptibility towards the trained concentration of the CS (Cho et al., 2016). Interestingly,

401 the modulated activity in the AWC ${ }^{\mathrm{ON}}$ neuron depended on intact neurotransmission (Suppl. Fig.

402 S11), demonstrating that network activity, rather than cell-autonomous processes, underlie

403 memory formation in this neuron.

405 valence of the experience. This was observed in the AWA neurons, and possibly also in the

$406 \mathrm{ASH}$ neurons (Fig. 2D-E). Thus, valence and stimulus components of the memory may be

407 distributed among individual sensory neurons, and integration of their modulated dynamics,

408 presumably, forms the associative memories. A division of encoding valence and the stimulus

409 components of the memory is also found in insects and mammalian brains (Gottfried et al.,

410 2002; Sacco and Sacchetti, 2010; Sekeres et al., 2010; Liu et al., 2012). It appears that

411 modularization of memory components is a fundamental principle that even compact nervous

412 systems adhere to.

413 These findings highlight the intriguing perspective that sensory systems are actively

414 participating to encode associative memories. In mammals, similar stimulus-specific

415 neuroplasticity was observed in primary sensory cortices (Morris, Friston and Dolan, 1998; Ohl

416 and Scheich, 2005) and in peripheral sensory neurons (Jones et al., 2008). Thus, 
417 neuroplasticity at the sensory system may have evolved as a universal solution to improve

418 detection and to enhance attention towards critical stimuli, thereby increasing animals' fitness

419 (McGann, 2015).

A limited set of chemosensory neurons was recruited to code each of the short-term

421 memories. Out of the 12 pairs of sensory neurons analyzed herein, only a single pair (AWC)

422 showed modulated activity following short-term positive training, and three pairs of neurons

423 (AWC, ASH, AWA) showed modulated activity following short-term aversive training (Fig. 2).

424 This activity sparseness may come as a surprise given the considerable connectivity among

425 the sensory neurons, either by direct wiring (chemical and electrical) or via feedback from

426 connected interneurons (White et al., 1986; Zaslaver et al., 2015; Cook et al., 2019).

427 Interestingly, associative learning in mice also led to sparse population coding, where the total

428 network activity was decreased while the response of cortical neurons was increased,

429 presumably to provide efficient sensory processing of the learned stimuli (Gdalyahu et al.,

430 2012).

431 The economic allocation of neural resources may point to an interesting design

432 evolved in animals with a compact neural network. Minimizing the number of neurons allocated

433 for encoding, and thus maintaining a sparse coding strategy, may maximize memory capacity

434 (Rolls and Treves, 1990). Furthermore, sensory neuroplasticity alters dynamic responses to

435 various environmental cues, and consequently, innate behavioral outputs may be modulated.

436 Thus, it may become advantageous to minimize the number of sensory neurons undergoing

437 functional changes so that a larger fraction of the sensory system can preserve innate

438 functions. In support of the minimal sensory units that can store a memory in C. elegans, the

439 AFD neurons had been shown to store the recently experienced temperature in a 440 cell-autonomous manner (Luo, Cook, et al., 2014; Tsukada et al., 2016). In addition, a few 
441 dedicated sensory neurons participate in learning and memory of salt concentrations (Luo,

442 Wen, et al., 2014; Jang et al., 2019).

444 sensory neurons, was observed in the immediate downstream layer of the interneurons (Fig.

445 3). We identified two interneurons, AIA and RIA, whose activity was modulated in a stimulus-

446 and valence- specific manner, both following short- and long-term learning. The AIA

447 interneurons are postsynaptic to most of the chemosensory neurons (White et al., 1986; Cook

448 et al., 2019), and had been shown to integrate various chemosensory inputs (Shinkai et al.,

449 2011; Larsch et al., 2015; Dobosiewicz, Liu and Bargmann, 2019). While AIA activity promotes

450 forward locomotion (Garrity et al., 2010), we found that long-term aversive training increased

451 AlA activity together with increase in reversal rates (Fig. 3D-F and Fig. 4E-F). This may

452 suggest that the AIA neurons are primarily integrating sensory modalities rather than directly

453 affecting motor outputs.

455 directionality, by integrating perceived sensory cues with motor-neuron feedback that signals

456 head position (Hendricks et al., 2012; Hendricks and Zhang, 2013; Ouellette et al., 2018). We

457 found that these neurons also store and integrate past stimuli and valence experiences (Figure

4583 G-L). Anatomically, the RIA neurons receive direct synaptic inputs from AWC and ASH, and

459 indirect inputs from AWA (White et al., 1986; Cook et al., 2019), possibly explaining how these

460 neurons may integrate memory information distributed within individual sensory neurons.

462 4G-H). The altered choice of direction (suppl. Fig. S15H) and the neuronal activity in RIA (Fig.

$4633 \mathrm{H})$ show correlating logic of regulation: both are markedly increased in LTAV. This might 464 suggest that the modulated activity in the RIAs may underlie the change in movement 465 directionality. In addition, RIA ablation mildly reduces reversal rates (Gray, Hill and Bargmann, 
466 2005), raising the possibility that these neurons also contribute to the enhanced turning

467 frequency and aversion in LTAV-trained worms (Fig. 4E-F).

469 (Wakabayashi, Kitagawa and Shingai, 2004; lino and Yoshida, 2009; Garrity et al., 2010; Li et

470 al., 2014; Lee et al., 2019), and speed (e.g., AIY, RIB, SIA, and RMG (Li et al., 2014; Lee et

471 al., 2019)), may also be participating in these memory-induced behavioral modulations.

472 Indeed, some of these neurons had been shown to participate in either memory formation (AIB

473 \& RIM) or memory retrieval (AIY \& RIA (Jin, Pokala and Bargmann, 2016)). In addition, while

474 we exclusively focused on neurons whose activity was modulated upon memory retrieval,

475 these and other neurons may contribute to memory formation via transcriptional changes

476 (Lakhina et al., 2015; Freytag et al., 2017). Together, interneurons might act as secondary

477 memory cells that integrate and store information from the upstream primary sensory neurons.

478 As activity of interneurons highly correlates with locomotion behavior (Kato et al., 2015), these

479 neurons directly dictate adaptive locomotion outputs upon encountering the CS.

481 markedly modified activity of the downstream interneurons (Fig. 3). This may hint to another

482 intriguing principle for coding memories in a compact neural network: Plasticity in the sensory

483 neurons is likely to modulate sensory responses to various cues, possibly affecting innate

484 behavioral outputs. For short-term memories, the modulated behavior will be brief, but for

485 long-term memories, the impact on behavior will be long lasting. Thus, for long-term memories,

486 it may be advantageous to 'clear up' information stored within sensory neurons, and to

487 relegate it to deeper layers of the network, so that animals could quickly resume innate

488 responses. This relegation of long-term memories to deeper layers can be viewed as

489 analogous to the transfer process taking place in mammalian brains, where hippocampal

490 short-term memories are moved for long-term storage in cortical areas (Rothschild, 2019). In 
491 addition, clearing information from sensory neurons may also mitigate the limited sensory

492 resources as more neurons will become available for coding new short-lived memories.

494 encoded within a compact neural network. The comprehensive cellular-resolution

495 network-wide analysis revealed numerous principles that allow efficient encoding under the

496 constraints of limited neural resources. These coding principles may well extend to memory

497 formation and storage in higher organisms with more complex brain systems.

499 Material and Methods section is available in the supplementary information file.

501 Data availability. All neuronal activity and behavioral data together with the associated

502 analysis scripts are available in $\underline{\text { https://osf.io/5v4qu/. }}$

503 Competing interests. The authors declare no competing interests.

\section{Acknowledgements}

505 We thank Cornelia Bargmann and Einav Gross for strains. Some strains were provided by the

506 CGC, which is funded by the NIH Office of Research Infrastructure Programs (P40

507 OD010440). This study was funded by ERC (336803), ICORE, and ISF (1300/17) to AZ. COP

508 postdoctoral fellowship is supported by the David-Herzog-Funds at Styrian Universities. EB,

$509 \mathrm{RR}$, and El are also supported by the Jerusalem Brain Center. AZ is the Joseph $\mathrm{H}$. and Belle

510 R. Braun Senior Lecture chair and the Greenfield chair in Neurobiology. 


\section{References}

512 Ahrens, M. B. et al. (2013) 'Whole-brain functional imaging at cellular resolution using

513 light-sheet microscopy', Nature methods, 10(5), pp. 413-420.

514 Åns, F. et al. (2013) 'Aversive learning increases sensory detection sensitivity', Biological

515 psychology, 92(2), pp. 135-141.

516 Aimon, S. et al. (2019) 'Fast near-whole-brain imaging in adult Drosophila during responses to

517 stimuli and behavior', PLOS Biology, p. e2006732. doi: 10.1371/journal.pbio.2006732.

518 Amano, H. and Maruyama, I. N. (2011) 'Aversive olfactory learning and associative long-term

519 memory in Caenorhabditis elegans', Learning \& memory , 18(10), pp. 654-665.

520 Ardiel, E. L. and Rankin, C. H. (2010) 'An elegant mind: learning and memory in

521 Caenorhabditis elegans', Learning \& memory, 17(4), pp. 191-201.

522 Bargmann, C. I., Hartwieg, E. and Horvitz, H. R. (1993) 'Odorant-selective genes and neurons

523 mediate olfaction in C. elegans', Cell, 74(3), pp. 515-527.

524 Byrne, J. H., Castellucci, V. F. and Kandel, E. R. (1978) 'Contribution of individual

525 mechanoreceptor sensory neurons to defensive gill-withdrawal reflex in Aplysia', Journal of

526 neurophysiology, 41(2), pp. 418-431.

527 Chalasani, S. H. et al. (2010) 'Neuropeptide feedback modifies odor-evoked dynamics in

528 Caenorhabditis elegans olfactory neurons', Nature Neuroscience, pp. 615-621. doi:

529 10.1038/nn.2526.

530 Cho, C. E. et al. (2016) 'Parallel encoding of sensory history and behavioral preference during

531 Caenorhabditis elegans olfactory learning', eLife. doi: 10.7554/elife.14000.

532 Chronis, N., Zimmer, M. and Bargmann, C. I. (2007) 'Microfluidics for in vivo imaging of

533 neuronal and behavioral activity in Caenorhabditis elegans', Nature methods, 4(9), pp.

534 727-731.

535 Cook, S. J. et al. (2019) 'Whole-animal connectomes of both Caenorhabditis elegans sexes',

536 Nature, 571(7763), pp. 63-71.

537 Dobosiewicz, M., Liu, Q. and Bargmann, C. I. (2019) 'Reliability of an interneuron response

538 depends on an integrated sensory state', eLife, 8. doi: 10.7554/eLife.50566.

539 Freytag, V. et al. (2017) 'Genome-Wide Temporal Expression Profiling in Caenorhabditis

540 elegans Identifies a Core Gene Set Related to Long-Term Memory', The Journal of

541 neuroscience: the official journal of the Society for Neuroscience, 37(28), pp. 6661-6672.

542 Garrity, P. A. et al. (2010) 'Running hot and cold: behavioral strategies, neural circuits, and the

543 molecular machinery for thermotaxis in C. elegans and Drosophila', Genes \& Development,

544 pp. 2365-2382. doi: 10.1101/gad.1953710.

545 Gdalyahu, A. et al. (2012) 'Associative fear learning enhances sparse network coding in

546 primary sensory cortex', Neuron, 75(1), pp. 121-132. 
547 Gottfried, J. A. et al. (2002) 'Functional Heterogeneity in Human Olfactory Cortex: An

548 Event-Related Functional Magnetic Resonance Imaging Study', The Journal of Neuroscience,

549 pp. 10819-10828. doi: 10.1523/jneurosci.22-24-10819.2002.

550 Gray, J. M., Hill, J. J. and Bargmann, C. I. (2005) 'A circuit for navigation in Caenorhabditis

551 elegans', Proceedings of the National Academy of Sciences, pp. 3184-3191. doi:

552 10.1073/pnas.0409009101.

553 Hendricks, M. et al. (2012) 'Compartmentalized calcium dynamics in a C. elegans interneuron

554 encode head movement', Nature, 487(7405), pp. 99-103.

555 Hendricks, M. and Zhang, Y. (2013) 'Complex RIA calcium dynamics and its function in

556 navigational behavior', Worm, 2(3), p. e25546.

557 Hopfield, J. J. (1982) 'Neural networks and physical systems with emergent collective

558 computational abilities', Proceedings of the National Academy of Sciences of the United

559 States of America, 79(8), pp. 2554-2558.

560 lino, Y. and Yoshida, K. (2009) 'Parallel use of two behavioral mechanisms for chemotaxis in

561 Caenorhabditis elegans', The Journal of neuroscience: the official journal of the Society for

562 Neuroscience, 29(17), pp. 5370-5380.

563 Itskovits, E. et al. (2017) 'A multi-animal tracker for studying complex behaviors', BMC biology, 564 15(1), p. 29.

565 Iwanir, S. et al. (2019) 'Irrational behavior in C. elegans arises from asymmetric modulatory 566 effects within single sensory neurons', Nature communications, 10(1), p. 3202.

567 Jang, M. S. et al. (2019) 'Multiple sensory neurons mediate starvation-dependent aversive

568 navigation inCaenorhabditis elegans', Proceedings of the National Academy of Sciences, pp.

569 18673-18683. doi: 10.1073/pnas.1821716116.

570 Jin, X., Pokala, N. and Bargmann, C. I. (2016) 'Distinct Circuits for the Formation and Retrieval

571 of an Imprinted Olfactory Memory', Cell, 164(4), pp. 632-643.

572 Jones, S. V. et al. (2008) 'Learning-Dependent Structural Plasticity in the Adult Olfactory

573 Pathway', Journal of Neuroscience, pp. 13106-13111. doi: 10.1523/jneurosci.4465-08.2008.

574 Josselyn, S. A. and Tonegawa, S. (2020) 'Memory engrams: Recalling the past and imagining

575 the future', Science, 367(6473). doi: 10.1126/science.aaw4325.

576 Kato, S. et al. (2014) 'Temporal Responses of C. elegans Chemosensory Neurons Are

577 Preserved in Behavioral Dynamics', Neuron, pp. 616-628. doi: 10.1016/j.neuron.2013.11.020.

578 Kato, S. et al. (2015) 'Global brain dynamics embed the motor command sequence of

579 Caenorhabditis elegans', Cell, 163(3), pp. 656-669.

580 Kauffman, A. et al. (2011) 'C. elegans positive butanone learning, short-term, and long-term

581 associative memory assays', Journal of visualized experiments: JoVE, (49). doi:

$58210.3791 / 2490$.

583 Kauffman, A. L. et al. (2010) 'Insulin signaling and dietary restriction differentially influence the

584 decline of learning and memory with age', PLoS biology, 8(5), p. e1000372.

585 Kerr, R. et al. (2000) 'Optical imaging of calcium transients in neurons and pharyngeal muscle 
586 of C. elegans', Neuron, 26(3), pp. 583-594.

587 Lakhina, V. et al. (2015) 'Genome-wide functional analysis of CREB/long-term

588 memory-dependent transcription reveals distinct basal and memory gene expression

589 programs', Neuron, 85(2), pp. 330-345.

590 Larsch, J. et al. (2015) 'A Circuit for Gradient Climbing in C. elegans Chemotaxis', Cell reports, 591 12(11), pp. 1748-1760.

592 Lee, J. B. et al. (2019) 'A compressed sensing framework for efficient dissection of neural 593 circuits', Nature methods, 16(1), pp. 126-133.

594 Liu, X. et al. (2012) 'Optogenetic stimulation of a hippocampal engram activates fear memory

595 recall', Nature, 484(7394), pp. 381-385.

$596 \mathrm{Li}, \mathrm{Z}$. et al. (2014) 'Encoding of Both Analog- and Digital-like Behavioral Outputs by One C.

597 elegans Interneuron', Cell, pp. 751-765. doi: 10.1016/j.cell.2014.09.056.

598 Luo, L., Cook, N., et al. (2014) 'Bidirectional thermotaxis in Caenorhabditis elegans is

599 mediated by distinct sensorimotor strategies driven by the AFD thermosensory neurons',

600 Proceedings of the National Academy of Sciences of the United States of America, 111(7),

601 pp. 2776-2781.

602 Luo, L., Wen, Q., et al. (2014) 'Dynamic Encoding of Perception, Memory, and Movement in a 603 C. elegans Chemotaxis Circuit', Neuron, pp. 1115-1128. doi: 10.1016/j.neuron.2014.05.010.

604 McGann, J. P. (2015) 'Associative learning and sensory neuroplasticity: how does it happen

605 and what is it good for?', Learning \& Memory, pp. 567-576. doi: 10.1101/Im.039636.115.

606 Morris, J. S., Friston, K. J. and Dolan, R. J. (1998) 'Experience-dependent modulation of 607 tonotopic neural responses in human auditory cortex', Proceedings of the Royal Society of 608 London. Series B: Biological Sciences, pp. 649-657. doi: 10.1098/rspb.1998.0343.

609 Nguyen, J. P. et al. (2016) 'Whole-brain calcium imaging with cellular resolution in freely 610 behaving Caenorhabditis elegans', Proceedings of the National Academy of Sciences of the 611 United States of America, 113(8), pp. E1074-81.

612 Ohl, F. W. and Scheich, H. (2005) 'Learning-induced plasticity in animal and human auditory 613 cortex', Current opinion in neurobiology, 15(4), pp. 470-477.

614 Ouellette, M.-H. et al. (2018) 'A Gate-and-Switch Model for Head Orientation Behaviors in

615 Caenorhabditis elegans', eneuro, pp. ENEURO.0121-18.2018. doi:

616 10.1523/eneuro.0121-18.2018.

617 Pavlov, I. P. (1910) The work of the digestive glands. C. Griffin.

618 Rolls, E. T. and Treves, A. (1990) 'The relative advantages of sparse versus distributed 619 encoding for associative neuronal networks in the brain', Network: Computation in Neural 620 Systems. Taylor \& Francis, 1(4), pp. 407-421.

621 Rothschild, G. (2019) 'The transformation of multi-sensory experiences into memories during

622 sleep', Neurobiology of learning and memory, 160, pp. 58-66.

623 Sacco, T. and Sacchetti, B. (2010) 'Role of secondary sensory cortices in emotional memory 
624 storage and retrieval in rats', Science, 329(5992), pp. 649-656.

625 Sasakura, H. and Mori, I. (2013) 'Behavioral plasticity, learning, and memory in C. elegans',

626 Current opinion in neurobiology, 23(1), pp. 92-99.

627 Schrödel, T. et al. (2013) 'Brain-wide 3D imaging of neuronal activity in Caenorhabditis

628 elegans with sculpted light', Nature methods, 10(10), pp. 1013-1020.

629 Sekeres, M. J. et al. (2010) 'Dorsal hippocampal CREB is both necessary and sufficient for

630 spatial memory', Learning \& memory , 17(6), pp. 280-283.

631 Shinkai, Y. et al. (2011) 'Behavioral Choice between Conflicting Alternatives Is Regulated by a

632 Receptor Guanylyl Cyclase, GCY-28, and a Receptor Tyrosine Kinase, SCD-2, in AIA

633 Interneurons of Caenorhabditis elegans', Journal of Neuroscience, pp. 3007-3015. doi:

634 10.1523/jneurosci.4691-10.2011.

635 Toyoshima, Y. et al. (2020) 'Neuron ID dataset facilitates neuronal annotation for whole-brain

636 activity imaging of C. elegans', BMC biology, 18(1), p. 30.

637 Tsukada, Y. et al. (2016) 'Reconstruction of Spatial Thermal Gradient Encoded in

638 Thermosensory Neuron AFD in Caenorhabditis elegans', The Journal of neuroscience: the

639 official journal of the Society for Neuroscience, 36(9), pp. 2571-2581.

640 Venkatachalam, V. et al. (2016) 'Pan-neuronal imaging in roaming Caenorhabditis elegans',

641 Proceedings of the National Academy of Sciences of the United States of America, 113(8),

642 pp. E1082-8.

643 Voleti, V. et al. (2019) 'Real-time volumetric microscopy of in vivo dynamics and large-scale

644 samples with SCAPE 2.0', Nature methods, 16(10), pp. 1054-1062.

645 Wakabayashi, T., Kitagawa, I. and Shingai, R. (2004) 'Neurons regulating the duration of

646 forward locomotion in Caenorhabditis elegans', Neuroscience research, 50(1), pp. 103-111.

647 White, J. G. et al. (1986) 'The structure of the nervous system of the nematode Caenorhabditis

648 elegans', Philosophical transactions of the Royal Society of London. Series B, Biological

649 sciences, 314(1165), pp. 1-340.

650 Widmann, A. et al. (2018) 'Odor-taste learning in Drosophila larvae', Journal of insect

651 physiology, 106(Pt 1), pp. 47-54.

652 Witvliet, D. et al. (2020) 'Connectomes across development reveal principles of brain

653 maturation in C. elegans', bioRxiv. biorxiv.org. Available at:

$654 \mathrm{https}: / /$ www.biorxiv.org/content/10.1101/2020.04.30.066209v1.abstract.

655 Zaslaver, A. et al. (2015) 'Hierarchical sparse coding in the sensory system of Caenorhabditis

656 elegans', Proceedings of the National Academy of Sciences of the United States of America,

657 112(4), pp. 1185-1189. 